\title{
Approximating the Schur multiplier of certain infinitely presented groups via nilpotent quotients
}

\author{
René Hartung
}

\begin{abstract}
We describe an algorithm for computing successive quotients of the Schur multiplier $M(G)$ of a group $G$ given by an invariant finite $L$-presentation. As applications, we investigate the Schur multipliers of various self-similar groups, including the Grigorchuk super-group, the generalized Fabrykowski-Gupta groups, the Basilica group and the Brunner-Sidki-Vieira group.
\end{abstract}

\section{Introduction}

The Schur multiplier $M(G)$ of a group $G$ can be defined as the second homology group $H_{2}(G, \mathbb{Z})$. It was introduced by Schur and is relevant, for instance, in the theory of central group extensions. In combinatorial group theory, the Schur multiplier found applications by virtue of the Hopf formula: if $F$ is a free group and $R$ is a normal subgroup of $F$ such that $G \cong F / R$, then the Schur multiplier of $G$ is isomorphic to the factor group $\left(R \cap F^{\prime}\right) /[R, F]$. For further details on the Schur multiplier we refer to [24, Chapter 11].

The Hopf formula yields that every finitely presentable group has a finitely generated Schur multiplier. This fact is used in [15] to prove that the Grigorchuk group is not finitely presentable, as its Schur multiplier is infinitely generated 2-elementary abelian, which answers the questions raised in $[\mathbf{6}, \mathbf{2 5}]$. Besides the Grigorchuk group, there are various examples of self-similar groups for which it is not known whether their Schur multiplier is finitely generated or whether the groups are finitely presented.

The first aim of this paper is to introduce an algorithm for investigating the Schur multiplier of a self-similar group, with a view towards demonstrating its finite generation. Let $G$ be a group with a presentation $G \cong F / R$. Then $G / \gamma_{c} G \cong F / R \gamma_{c} F$, where $\gamma_{c} G$ is the $c$ th term of the lower central series of $G$. We identify $M(G)$ with $\left(R \cap F^{\prime}\right) /[R, F]$ and $M\left(G / \gamma_{c} G\right)$ with $\left(R \gamma_{c} F \cap F^{\prime}\right) /\left[R \gamma_{c} F, F\right]$ and define

$$
\varphi_{c}: M(G) \rightarrow M\left(G / \gamma_{c} G\right), \quad g[R, F] \mapsto g\left[R \gamma_{c} F, F\right] .
$$

Then $\varphi_{c}$ is a homomorphism of abelian groups. We describe an effective method to determine the Dwyer quotients $M_{c}(G)=M(G) / \operatorname{ker} \varphi_{c}$ for $c \in \mathbb{N}$, provided that $G$ is given by an invariant finite $L$-presentation; see $[\mathbf{1}, \mathbf{2}]$ or Section 2 below. Every finitely presented group and many selfsimilar groups can be described by a finite invariant $L$-presentation. An implementation of our algorithm is available in the NQL package [19] of the computer algebra system GAP; see [12].

We have applied our algorithm to various examples of self-similar groups: the Grigorchuk super-group $\mathfrak{\mathfrak { G }}$ (see $[\mathbf{3}]$ ), the Basilica group $\Delta$ (see [17, 18]), the Brunner-Sidki-Vieira group BSV (see $[\mathbf{9}]$ ) and some generalized Fabrykowski-Gupta groups $\Gamma_{d}$ (see $[\mathbf{1 1}, \mathbf{1 6}]$ ). We found that the sequence $\left(M_{1}(G), \ldots, M_{c}(G), M_{c+1}(G), \ldots\right)$ exhibits a periodicity in $c$ in all these cases. Based on this observation, we propose the following conjecture.

\section{Conjecture I.}

- For $c \geqslant 4, M_{c}(\tilde{\mathfrak{G}})$ is 2-elementary abelian of rank $2\left\lfloor\log _{2}(c)\right\rfloor+2\left\lfloor\log _{2}(c / 3)\right\rfloor+5$. 
- For $c \geqslant 6, M_{c}(\Delta)$ has the form $\mathbb{Z}^{2} \times \mathcal{A}_{c}$, where $\mathcal{A}_{c}$ is an abelian 2-group of rank $\left\lfloor\log _{2}(c / 3)\right\rfloor$ and exponent $2^{2\lfloor(c-6) / 2\rfloor+2}$.

- For $c \geqslant 4, M_{c}(\mathrm{BSV})$ has the form $\mathbb{Z}^{2} \times \mathcal{B}_{c}$, where $\mathcal{B}_{c}$ is an abelian 2-group of rank $\left\lfloor\log _{2}(c / 5)\right\rfloor+\left\lfloor\log _{2}(c / 9)\right\rfloor+3$ and exponent $2^{2\lfloor(c-4) / 2\rfloor+1}$.

- For a prime power $d$, the group $M_{c}\left(\Gamma_{d}\right)$ has exponent $d$ for $c$ large enough; its rank is an increasing function of $c$ which exhibits a periodic pattern.

In particular, all of these groups have an infinitely generated Schur multiplier and are therefore not finitely presentable.

Further details on the periodicities and the computational evidence for them are given in Section 6 .

\section{Preliminaries}

In this section, we recall the basic notion of invariant and finite $L$-presentations and the basic theory of the Schur multiplier of a group. Let $F$ be a finitely generated free group over the alphabet $\mathcal{X}$. Further, suppose that $\mathcal{Q}, \mathcal{R} \subset F$ are finite subsets of the free group $F$ and that $\Phi \subset \operatorname{End}(F)$ is a finite set of endomorphisms of $F$. Then the quadruple $\langle\mathcal{X}|\mathcal{Q}| \Phi \mid \mathcal{R}\rangle$ is a finite $L$-presentation. It defines the finitely $L$-presented group

$$
G=\left\langle\mathcal{X} \mid \mathcal{Q} \cup \bigcup_{\varphi \in \Phi^{*}} \mathcal{R}^{\varphi}\right\rangle,
$$

where $\Phi^{*}$ denotes the free monoid generated by $\Phi$, that is, the closure of $\Phi \cup\{$ id $\}$ under composition. A finite $L$-presentation $\langle\mathcal{X}|\mathcal{Q}| \Phi \mid \mathcal{R}\rangle$ is invariant if every endomorphism $\varphi \in \Phi$ induces an endomorphism of $G$, that is, if the normal closure of $\mathcal{Q} \cup \bigcup_{\varphi \in \Phi^{*}} \mathcal{R}^{\varphi}$ in $F$ is $\varphi$-invariant. For example, every finite $L$-presentation of the form $\langle\mathcal{X}|\emptyset| \Phi \mid \mathcal{R}\rangle$ is invariant. Clearly, invariant finite $L$-presentations generalize finite presentations, since every finitely presented group $\langle\mathcal{X} \mid \mathcal{R}\rangle$ is finitely $L$-presented by $\langle\mathcal{X}|\emptyset|\{$ id $\} \mid \mathcal{R}\rangle$. Further examples of invariantly $L$-presented groups include self-similar groups such as the Grigorchuk group [14], the Basilica group $[\mathbf{1 7}, \mathbf{1 8}]$ and the Brunner-Sidki-Vieira group [9].

In the remainder of this section, we recall the basic theory of the Schur multiplier of a group $G$. Recall that, in general, the Schur multiplier of a finitely presented group is not computable; see [13]. But if, for instance, $G$ is finite, then $M(G)$ can be deduced from a finite presentation of $G$ by means of the Hopf formula and the Reidemeister-Schreier algorithm. A more effective algorithm for finite permutation groups is described in [20]. Recently, Eick and Nickel [10] described an algorithm for computing the Schur multiplier of a polycyclic group given by a polycyclic presentation.

Let $F$ be a free group and let $R$ be a normal subgroup of $F$ such that $G \cong F / R$. Then the Hopf formula gives

$$
M(G) \cong\left(R \cap F^{\prime}\right) /[R, F] .
$$

Suppose that $N$ is a normal subgroup of $G$, and let $S$ be a normal subgroup of $F$ such that $S R / R$ corresponds to $N$. Then Blackburn and Evens [7] determined the exact sequence

$$
1 \rightarrow(R \cap[S, F]) /([R, F] \cap[S, F]) \rightarrow M(G) \rightarrow M(G / N) \rightarrow\left(N \cap G^{\prime}\right) /[N, G] \rightarrow 1 .
$$

Applying this sequence to the lower central series term $N=\gamma_{c} G$ yields the exact sequence

$$
1 \rightarrow\left(R \cap \gamma_{c+1} F\right) /\left([R, F] \cap \gamma_{c+1} F\right) \rightarrow M(G) \stackrel{\varphi_{c}}{\rightarrow} M\left(G / \gamma_{c} G\right) \rightarrow \gamma_{c} G / \gamma_{c+1} G \rightarrow 1
$$

This gives a filtration $M(G) \geqslant \operatorname{ker} \varphi_{1} \geqslant \operatorname{ker} \varphi_{2} \geqslant \ldots$, called the Dwyer filtration, of the Schur multiplier of $G$. Note that if $G$ has a maximal nilpotent quotient of class $c$, then

$$
\bigcap_{c \in \mathbb{N}_{0}} \operatorname{ker} \varphi_{c} \cong\left(R \cap \gamma_{c+1} F\right)[R, F] /[R, F]
$$


However, even if the group $G$ is residually nilpotent, the group $F /[R, F]$ is not necessarily residually nilpotent; see $[\mathbf{8 , 2 2}]$. Thus, the $D$ wyer kernel $\bigcap_{c \in \mathbb{N}} \operatorname{ker} \varphi_{c}$ is possibly non-trivial.

We note that the Schur multiplier $M\left(G / \gamma_{c} G\right)$ can be computed by using the algorithm in [10], while the isomorphism type of $\gamma_{c} G / \gamma_{c+1} G$ can be computed with the nilpotent quotient algorithm in [2]. Therefore, the sequence $M(G) \rightarrow M\left(G / \gamma_{c} G\right) \rightarrow \gamma_{c} G / \gamma_{c+1} G \rightarrow 1$ allows us to determine the size of $M_{c}(G)$ provided that $M\left(G / \gamma_{c} G\right)$ is finite. On the other hand, the algorithm described here determines the structure of $M_{c}(G)$ even when the Schur multiplier $M\left(G / \gamma_{c} G\right)$ is infinite.

\section{Adjusting an invariant $L$-presentation}

In order to prove the following theorem, we explicitly describe an algorithm for modifying an invariant $L$-presentation. The resulting $L$-presentation enables us to read off a generating set for the Schur multiplier in Section 4. Our algorithm generalizes the explicit computations in [15].

Theorem 1. Let $\langle\mathcal{X}|\mathcal{Q}| \Phi \mid \mathcal{R}\rangle$ be an invariant finite L-presentation which defines the group $G=F / R$. Then $G$ admits an invariant finite $L$-presentation $\left\langle\mathcal{X}\left|\mathcal{Q}^{\prime} \cup \mathcal{B}\right| \Phi \mid \mathcal{R}^{\prime}\right\rangle$ such that $\mathcal{Q}^{\prime}, \mathcal{R}^{\prime} \subset F^{\prime}$ and $\mathcal{B} \subset F$ satisfies $|\mathcal{B}|=|\mathcal{X}|-\mathfrak{h}\left(G / G^{\prime}\right)$, where $\mathfrak{h}\left(G / G^{\prime}\right)$ denotes the torsion-free rank of $G / G^{\prime}$.

Proof. Since $\langle\mathcal{X}|\mathcal{Q}| \Phi \mid \mathcal{R}\rangle$ is an invariant $L$-presentation, every endomorphism $\varphi \in \Phi$ induces an endomorphism of the group $G$. Thus we have $R^{\varphi} \subseteq R$ for every $\varphi \in \Phi^{*}$. In particular, every image of a relator in $\mathcal{Q} \cup \mathcal{R}$ is a consequence, that is, $\mathcal{Q}^{\varphi} \subset R$ and $\mathcal{R}^{\varphi} \subset R$ for every $\varphi \in \Phi^{*}$.

Write $n=\operatorname{rk}(F)$. Then the abelianization $\pi: F \rightarrow \mathbb{Z}^{n}$ maps each $x \in F$ to its corresponding exponent vector $a_{x} \in \mathbb{Z}^{n}$. Clearly, ker $\pi=F^{\prime}$ and, since $F^{\prime}$ is fully invariant, every $\varphi \in \Phi$ induces an endomorphism of the free abelian group $\mathbb{Z}^{n}$. Therefore, the exponent vector of $x^{\varphi}$ is the image $a_{x} M_{\varphi}$ for some matrix $M_{\varphi} \in \mathbb{Z}^{n \times n}$. Now, the normal subgroup $R F^{\prime}$ maps onto

$$
U=\left\langle a_{q}, a_{r} M_{\varphi} \mid q \in \mathcal{Q}, r \in \mathcal{R}, \varphi \in \Phi^{*}\right\rangle \leqslant \mathbb{Z}^{n} .
$$

As every subgroup of $\mathbb{Z}^{n}$ is generated by at most $n$ elements, the subgroup $U$ is finitely generated. In the following, we use the spinning algorithm from [2] along with Hermite normal form computations to compute a basis for the subgroup $U$ while modifying the $L$-presentation simultaneously.

Let $B$ be a basis of $\left\langle a_{q} \mid q \in \mathcal{Q}\right\rangle$. Then every element $u \in B$ is a $\mathbb{Z}$-linear combination of elements in $\left\{a_{q} \mid q \in \mathcal{Q}\right\}$. Hence, for each $u \in B$, there exists a word $r_{u}$ in the relators in $\mathcal{Q}$ such that $a_{r_{u}}=u$. Define $\mathcal{B}=\left\{r_{u} \mid u \in B\right\}$. Then for every $q \in \mathcal{Q}$, we have $a_{q} \in\langle B\rangle$ since $B$ is a basis, and hence there exists a word $w_{q}$ in the $r_{u}$ such that $a_{w_{q}}=a_{q}$. Define $\mathcal{Q}^{\prime}=\left\{q w_{q}^{-1} \mid q \in \mathcal{Q}\right\}$. Then the exponent vector of each element in $\mathcal{Q}^{\prime}$ vanishes, and hence $\mathcal{Q}^{\prime} \subset F^{\prime}$. Moreover, the invariant and finite $L$-presentation

$$
\left\langle\mathcal{X}\left|\mathcal{Q}^{\prime} \cup \mathcal{B}\right| \Phi \mid \mathcal{R}\right\rangle
$$

still defines the group $G$, since we only applied Tietze transformations to the given $L$-presentation.

It remains to force the elements of $\mathcal{R}$ into the derived subgroup $F^{\prime}$. For this purpose, we will use the spinning algorithm from [2], as follows. Initialize $\mathcal{R}^{\prime}=\emptyset$. As long as $\mathcal{R}$ is nonempty, take an element $r \in \mathcal{R}$ and remove it from $\mathcal{R}$. Then either $a_{r} \in\langle B\rangle$ or $a_{r} \notin\langle B\rangle$ holds. If $a_{r} \in\langle B\rangle$, then there exists a word $w_{r}$ in the $r_{u}$ such that $a_{w_{r}}=a_{r}$ and hence $r w_{r}^{-1} \in F^{\prime}$. In this case, we just add $r w_{r}^{-1}$ to $\mathcal{R}^{\prime}$. Note that for every $\varphi \in \Phi^{*}$, the word $\left(w_{r}^{-1}\right)^{\varphi}$ is a consequence and hence we can replace the relator $r^{\varphi}$ in the $L$-presentation by $\left(r w_{r}^{-1}\right)^{\varphi}$. The invariant and finite $L$-presentation

$$
\left\langle\mathcal{X}\left|\mathcal{Q}^{\prime} \cup \mathcal{B}\right| \Phi \mid \mathcal{R}^{\prime} \cup \mathcal{R}\right\rangle
$$

still defines the group $G$. 
On the other hand, if $a_{r} \notin\langle B\rangle$ holds, we enlarge the current basis $B$ and modify the set $\mathcal{B}$. Let $B^{\prime}$ be a basis for $\left\langle B \cup\left\{a_{r}\right\}\right\rangle$. Then every $v \in B^{\prime}$ is a $\mathbb{Z}$-linear combination of the elements in $B \cup\left\{a_{r}\right\}$, and hence there exists a word $\tilde{r}_{v}$ in $\mathcal{B} \cup\{r\}$ such that $a_{\tilde{r}_{v}}=v$. Define $\mathcal{B}=\left\{\tilde{r}_{v} \mid v \in B^{\prime}\right\}$. Then, by construction, either $|\mathcal{B}|=|B|+1$ or $|\mathcal{B}|=|B|$ holds. In the latter case, there is an element $u \in B$ such that $u \in\left\langle(B \backslash\{u\}) \cup\left\{a_{r}\right\}\right\rangle$ holds. Thus, there exists a word $w_{u}$ in the elements of $\mathcal{B}$ such that $a_{w_{u}}=u$ and hence $r_{u} w_{u}^{-1} \in F^{\prime}$. In this case, we add $r_{u} w_{u}^{-1}$ to $\mathcal{Q}^{\prime}$ and add the images $\left\{r_{u}^{\varphi} \mid \varphi \in \Phi\right\}$ to $\mathcal{R}$. This yields an invariant and finite $L$-presentation $\left\langle\mathcal{X}\left|\mathcal{Q}^{\prime} \cup \mathcal{B}\right| \Phi \mid \mathcal{R}^{\prime} \cup \mathcal{R}\right\rangle$, with $\mathcal{Q}^{\prime}, \mathcal{R}^{\prime} \subset F^{\prime}$, which still defines the group $G$.

Because ascending chains of subgroups in $\mathbb{Z}^{n}$ terminate, eventually every exponent vector of an element in $\mathcal{R}$ will be contained in the subgroup $\langle B\rangle$, and hence the algorithm described above must eventually terminate. Clearly, the basis $B$ is then a basis for the subgroup $U$ in (2). As shown in [2], the abelian quotient $G / G^{\prime}$ is isomorphic to the factor $\mathbb{Z}^{n} / U$. Its torsion-free rank is $n-|B|$ as claimed above.

In the following example, we recall the explicit computations in [15] for the Grigorchuk group $\mathfrak{G}$.

EXAMPLE 2. Consider the Grigorchuk group $\mathfrak{G}$ with its invariant $L$-presentation

$$
\left.\mathfrak{G} \cong\left\langle\{a, b, c, d\}\left|\left\{a^{2}, b^{2}, c^{2}, d^{2}, b c d\right\}\right|\{\sigma\}\right|\left\{(a d)^{4},(\text { adacac })^{4}\right\}\right\rangle,
$$

where $\sigma$ is the free group endomorphism induced by the mapping

$$
\sigma:\left\{\begin{array}{l}
a \mapsto c^{a}, \\
b \mapsto d, \\
c \mapsto b, \\
d \mapsto c .
\end{array}\right.
$$

The exponent vectors $(2,0,0,0),(0,1,1,1),(0,0,2,0)$ and $(0,0,0,2)$ of the relations $a^{2}, b c d$, $c^{2}$ and $d^{2}$, respectively, are $\mathbb{Z}$-linearly independent and form a basis for the subgroup $U$ in (2); therefore we can modify the above presentation so that the relations become

$$
a^{2}, c^{2}, d^{2}, b c d, b^{2}(b c d)^{-2} c^{2} d^{2}, \sigma^{k}\left((a d)^{4} a^{-4} d^{-4}\right), \sigma^{k}\left((a d a c a c)^{4} a^{-12} c^{-8} d^{-4}\right)
$$

for $k \in \mathbb{N}_{0}$. Since the $L$-presentation is invariant, the images $\sigma^{k}\left(a^{-4} d^{-4}\right)$ and $\sigma^{k}\left(a^{-12} c^{-8} d^{-4}\right)$ are consequences. Hence, the invariant finite $L$-presentation

$$
\left\langle\{a, b, c, d\}\left|\left\{b^{2}(b c d)^{-2} c^{2} d^{2}\right\} \cup\left\{a^{2}, c^{2}, d^{2}, b c d\right\}\right|\{\sigma\} \mid \mathcal{R}^{\prime}\right\rangle,
$$

where $\mathcal{R}^{\prime}=\left\{(a d)^{4} a^{-4} d^{-4},(\text { adacac })^{4} a^{-12} c^{-8} d^{-4}\right\}$, defines the Grigorchuk group $\mathfrak{G}$, and, since $\mathfrak{G} / \mathfrak{G}^{\prime} \cong \mathbb{Z}_{2}^{3}$, it has the form asserted in Theorem 1 .

\section{A generating set for the Schur multiplier}

Let $G$ be a finitely generated group. We will use the results of Theorem 1 and the Hopf formula to give a generating set for the Schur multiplier of $G$ in the case where $G$ is invariantly finitely $L$-presented. Suppose that $F$ is a finitely generated free group and that $R$ is a normal subgroup of $F$ such that $G \cong F / R$. Then $F /[R, F]$ is a central extension of $R /[R, F]$ by the group $G$, and the subgroup $R /[R, F]$ contains $\left(R \cap F^{\prime}\right) /[R, F]$. By the Hopf formula, the latter subgroup is isomorphic to the Schur multiplier of $G$. Furthermore, the subgroup $R /[R, F]$ decomposes as follows.

Proposition 1. Let $G \cong F / R$ with a finitely generated free group $F$. Then

$$
R /[R, F] \cong \mathbb{Z}^{\mathrm{rk}(F)-\mathfrak{h}\left(G / G^{\prime}\right)} \oplus M(G)
$$


Proof. The factor $R F^{\prime} / F^{\prime}$ is free abelian with torsion-free $\operatorname{rank} \operatorname{rk}(F)-\mathfrak{h}\left(G / G^{\prime}\right)$. Since $R F^{\prime} / F^{\prime} \cong R /\left(R \cap F^{\prime}\right)$ is free abelian, the subgroup $\left(R \cap F^{\prime}\right) /[R, F]$ has a free abelian complement of $\operatorname{rank} \operatorname{rk}(F)-\mathfrak{h}\left(G / G^{\prime}\right)$, and thus the central subgroup $R /[R, F]$ decomposes as claimed.

As $R /[R, F]$ is central in $F /[R, F]$, it is generated by the images of the normal generators of $R$. Thus, in particular, if $R$ is finitely generated as a normal subgroup (that is, if $G$ is finitely presentable), then $R /[R, F]$ is a finitely generated abelian group and so is its subgroup $\left(R \cap F^{\prime}\right) /[R, F]$.

If $G$ is finite, then $R /[R, F]$ is an abelian subgroup with finite index in $F /[R, F]$. A finite presentation for $F /[R, F]$ can be obtained from a finite presentation of $G$. Then the Reidemeister-Schreier algorithm yields a finite presentation for $R /[R, F]$, from which the isomorphism type of $M(G)$ is obtained easily.

If $G$ is polycyclic, then it is finitely presentable, and hence the group $F /[R, F]$ is an extension of a finitely generated abelian group by a polycyclic group. In particular, $F /[R, F]$ is polycyclic in this case. A consistent polycyclic presentation for $F /[R, F]$ can be computed with the algorithm in [10]. This polycyclic presentation enables us to read off the isomorphism type of $R /[R, F]$ and, by Proposition 1, the isomorphism type of $M(G)$. If $G$ is finitely generated and nilpotent of class $c$, then $F /[R, F]$ is nilpotent of class at most $c+1$. If $G$ is given by a weighted nilpotent presentation, then the algorithm in [23] computes a weighted nilpotent presentation for $F /[R, F]$.

We now consider the case of an invariantly finitely $L$-presented group $G$. Even though its Schur multiplier is not computable in general, the following theorem yields a generating set for $M(G)$ as a subgroup of $R /[R, F]$.

Theorem 3. Let $\left\langle\mathcal{X}\left|\mathcal{Q}^{\prime} \cup \mathcal{B}\right| \Phi \mid \mathcal{R}^{\prime}\right\rangle$ be an invariant finite $L$-presentation of $G$ as provided by Theorem 1. Further, let $\pi: F \rightarrow F /[R, F], x \mapsto \bar{x}$ denote the natural homomorphism. Then

$$
M(G) \cong\left\langle\bar{q}, \overline{r^{\varphi}} \mid q \in \mathcal{Q}^{\prime}, r \in \mathcal{R}^{\prime}, \varphi \in \Phi^{*}\right\rangle .
$$

Proof. Clearly, $R /[R, F]$ is generated by the images of $\mathcal{Q}^{\prime} \cup \mathcal{B} \cup \bigcup_{\varphi \in \Phi^{*}}\left(\mathcal{R}^{\prime}\right)^{\varphi}$. As the relators in $\mathcal{Q}^{\prime} \cup \mathcal{R}^{\prime}$ are contained in $F^{\prime}$, we have

$$
\left\{\bar{q}, \overline{r^{\varphi}} \mid q \in \mathcal{Q}^{\prime}, r \in \mathcal{R}^{\prime}, \varphi \in \Phi^{*}\right\} \subseteq\left(R \cap F^{\prime}\right) /[R, F] .
$$

We are left with the relators in $\mathcal{B}$. Recall that $|\mathcal{B}|=\operatorname{rk}(F)-\mathfrak{h}\left(G / G^{\prime}\right)$. Hence, the images $\{\bar{r} \mid r \in \mathcal{B}\}$ generate a free abelian complement to the Schur multiplier $\left(R \cap F^{\prime}\right) /[R, F]$ in $R /[R, F]$. Therefore, the images in (4) necessarily generate $\left(R \cap F^{\prime}\right) /[R, F]$.

As the group $G$ in Theorem 3 is invariantly $L$-presented, for every endomorphism $\varphi \in \Phi$ we have $R^{\varphi} \subseteq R$ and $[R, F]^{\varphi} \subseteq[R, F]$. Therefore, every $\varphi \in \Phi$ also induces an endomorphism of $F /[R, F]$ which fixes the subgroup $R /[R, F]$. Further, as $F^{\prime}$ is fully invariant, every such $\varphi$ induces an endomorphism $\bar{\varphi}$ of $\left(R \cap F^{\prime}\right) /[R, F]$. This yields

$$
M(G) \cong\left\langle\bar{q}, \bar{r} \bar{\varphi} \mid q \in \mathcal{Q}^{\prime}, r \in \mathcal{R}^{\prime}, \varphi \in \Phi^{*}\right\rangle,
$$

and hence the free monoid $\Phi^{*}$ induces a $\Phi^{*}$-module structure on the Schur multiplier $M(G)$ in a natural way.

Lemma 4. Let $\langle\mathcal{X}|\mathcal{Q}| \Phi \mid \mathcal{R}\rangle$ be an invariant finite L-presentation. Then the Schur multiplier $M(G)$ is finitely generated as a $\Phi^{*}$-module.

In particular, the Schur multiplier $M(G)$ has the form $A \oplus \bigoplus_{\Phi^{*}} B$ with finitely generated abelian groups $A$ and $B$; see [1]. 
We proceed further with Example 2 by describing a generating set for the Schur multiplier of the Grigorchuk group as provided by Theorem 3; cf. [15].

EXAmple 5. Consider the invariant finite $L$-presentation of the Grigorchuk group $\mathfrak{G}$ as determined in Example 2. Then the images of

$$
b^{2}(b c d)^{-2} c^{2} d^{2}, \sigma^{k}\left((a d)^{4} a^{-4} d^{-4}\right), \sigma^{k}\left((a d a c a c)^{4} a^{-12} c^{-8} d^{-4}\right) \quad \text { for } k \in \mathbb{N}_{0}
$$

in $F /[R, F]$ generate the subgroup $\left(R \cap F^{\prime}\right) /[R, F]$. The images in $F /[R, F]$ of the relations $a^{2}, c^{2}, d^{2}$ and $b c d$ generate a free abelian complement to the Schur multiplier $\left(R \cap F^{\prime}\right) /[R, F]$ in $R /[R, F]$.

\section{Approximating the Schur multiplier}

Now we describe our algorithm for approximating the Schur multiplier of an invariantly finitely $L$-presented group $G$. Let $\langle\mathcal{X}|\mathcal{Q}| \Phi \mid \mathcal{R}\rangle$ be an invariant finite $L$-presentation defining the group $F / R$ such that $G \cong F / R$. Then $G$ is finitely generated and hence its lower central series quotient $G / \gamma_{c} G$ is polycyclic. The nilpotent quotient algorithm in [2] computes a weighted nilpotent presentation for $G / \gamma_{c} G$ together with the natural homomorphism $\pi: F \rightarrow G / \gamma_{c} G$. In [23], Nickel described a covering algorithm which, given a weighted nilpotent presentation for $G / \gamma_{c} G$ and the homomorphism $\pi$, computes a polycyclic presentation for $F /\left[R \gamma_{c} F, F\right]$ together with the natural homomorphism $\bar{\pi}: F \rightarrow F /\left[R \gamma_{c} F, F\right]$. The homomorphism $\bar{\pi}$ induces the homomorphism $\varphi_{c}: M(G) \rightarrow M\left(G / \gamma_{c} G\right)$ as follows. By Theorem 1, the group $G$ has an invariant finite $L$-presentation of the form

$$
\left\langle\mathcal{X}\left|\mathcal{Q}^{\prime} \cup \mathcal{B}\right| \Phi \mid \mathcal{R}^{\prime}\right\rangle \quad \text { with } \mathcal{Q}^{\prime}, \mathcal{R}^{\prime} \subset F^{\prime} \text { and }|\mathcal{B}|=|\mathcal{X}|-\mathfrak{h}\left(G / G^{\prime}\right) .
$$

Now, by Theorem 3, the images of $\mathcal{Q}^{\prime} \cup \bigcup_{\varphi \in \Phi^{*}}\left(\mathcal{R}^{\prime}\right)^{\varphi}$ in $F /[R, F]$ generate the subgroup $\left(R \cap F^{\prime}\right) /[R, F]$. Similarly, their images in the factor group $F /\left[R \gamma_{c} F, F\right]$ generate the subgroup $\left(R \cap F^{\prime}\right)\left[R \gamma_{c} F, F\right] /\left[R \gamma_{c} F, F\right]$. Since $\left[R \gamma_{c} F, F\right]=[R, F] \gamma_{c+1} F$, we have

$$
\left(R \cap F^{\prime}\right)\left[R \gamma_{c} F, F\right] /\left[R \gamma_{c} F, F\right]=\left(R \gamma_{c+1} F \cap F^{\prime}\right) /\left[R \gamma_{c} F, F\right] .
$$

The latter subgroup is contained in $\left(R \gamma_{c} F \cap F^{\prime}\right) /\left[R \gamma_{c} F, F\right]$, which is isomorphic to the Schur multiplier $M\left(G / \gamma_{c} G\right)$.

As the group $G$ is invariantly $L$-presented, each $\varphi \in \Phi$ induces an endomorphism $\tilde{\varphi}$ of $R \gamma_{c} F /\left[R \gamma_{c} F, F\right]$, which yields that the image of $M(G)$ in $M\left(G / \gamma_{c} G\right)$ has the form

$$
\left\langle q^{\bar{\pi}},\left(r^{\bar{\pi}}\right)^{\tilde{\varphi}} \mid q \in \mathcal{Q}^{\prime}, r \in \mathcal{R}^{\prime}, \varphi \in \Phi^{*}\right\rangle .
$$

This can be used to investigate the $\Phi^{*}$-module structure of $M(G)$ by considering the finitely generated Dwyer quotients $M_{c}(G)$. In our algorithm, we use Hermite normal form computations in a spinning algorithm to compute a finite generating set of the subgroup in (6). Our algorithm is summarized as follows, where $G=F / R$.

\section{DWYERQuotient $(G, c)$}

Compute an invariant finite $L$-presentation as in Theorem 1.

Compute a weighted nilpotent presentation for $G / \gamma_{c} G$ together with the natural homomorphism $F \rightarrow G / \gamma_{c} G$.

Compute a polycyclic presentation for the group $F /\left[R \gamma_{c} F, F\right]$ together with the natural homomorphism $F \rightarrow F /\left[R \gamma_{c} F, F\right]$.

Translate each $\varphi \in \Phi$ to an endomorphism of the group $F /\left[R \gamma_{c} F, F\right]$ and restrict this endomorphism to $\left(R \gamma_{c+1} F \cap F^{\prime}\right) /\left[R \gamma_{c} F, F\right]$.

Use the spinning algorithm to compute a finite generating set for the image $\left(R \gamma_{c+1} F \cap F^{\prime}\right) /\left[R \gamma_{c} F, F\right]$. 


\section{Applications}

The algorithm described above is available in the NQL package [19] of the computer algebra system GAP (see [12]). We parallelized the algorithm in [2] to enlarge the possible depths in the lower central series reached in this section. We demonstrate the successful application of our algorithm to the following invariantly finitely $L$-presented testbed groups studied in $[\mathbf{1}, \mathbf{2}]$ :

- the Grigorchuk group $\mathfrak{G}$ (see [14]), with its invariant finite $L$-presentation from [21] (see also [15] and Example 2);

- the twisted twin $\overline{\mathfrak{G}}$ of the Grigorchuk group (see [4]), with its invariant finite $L$-presentation from $[4]$;

- the Grigorchuk super-group $\tilde{\mathfrak{G}}$ (see [3]), with its invariant finite $L$-presentation from [1];

- the Basilica group $\Delta$ (see $[\mathbf{1 7}, \mathbf{1 8}]$ ), with its invariant finite $L$-presentation from [5]; and

- the Brunner-Sidki-Vieira group BSV (see [9]), with its invariant finite $L$-presentation from $[\mathbf{1}]$.

In $\S 6.3$, we further apply our algorithm to several generalized Fabrykowski-Gupta groups, an infinite family of finitely $L$-presented groups $\Gamma_{p}$ introduced in [16]. Invariant finite $L$-presentations for these groups were computed in [2].

\subsection{Aspects of the implementation of our algorithm in GAP}

Table 1 shows some performance data relating to the implementation of our algorithm in the NQL package of the computer algebra system GAP. All timings displayed below were obtained on an Intel Pentium Core 2 Quad processor with clock speed $2.83 \mathrm{GHz}$ using a single core. For each application of our algorithm, we imposed a time limit of two hours; the computations were then stopped, and the total time taken to compute a weighted nilpotent presentation for the quotient $G / \gamma_{c} G$ as well as the total time to compute the Dwyer quotient $M_{c}(G)$ were recorded. Every application of the algorithm completed within 1 GB of memory.

We note that the results reported in the remainder of this section were obtained from using a parallel version of the algorithm for computing $G / \gamma_{c+1} G$.

\subsection{Dwyer quotients of the testbed groups}

The Dwyer quotient $M_{c}(G)=M(G) / \operatorname{ker} \varphi_{c}$ is a finitely generated abelian group; hence it can be described by its abelian invariants or, if the group is $p$-elementary abelian, by its $p$-rank. Here the list $\left(c_{1}, \ldots, c_{n}\right)$ stands for the group $\mathbb{Z}_{c_{1}} \oplus \ldots \oplus \mathbb{Z}_{c_{n}}$. We shall use the abbreviation $a^{[\ell]}$ to mean that the term $a$ occurs in $\ell$ consecutive places in a list. In the following, we summarize our computational results for the testbed groups.

The Grigorchuk group $\mathfrak{G}$ was shown in $[\mathbf{1 4}]$ to be an explicit counter-example to the general Burnside problem: it is a finitely generated infinite 2-torsion group. Furthermore, the Grigorchuk group is one of the first examples of a group with an intermediate word-growth.

TABLE 1. Performance data on our algorithm's implementation in GAP.

\begin{tabular}{cccc}
\hline & & \multicolumn{2}{c}{ Time (h:min) for } \\
\cline { 3 - 4 }$G$ & $c$ & $G / \gamma_{c+1} G$ & $M_{c+1}(G)$ \\
\hline $\mathfrak{G}$ & 90 & $1: 47$ & $0: 07$ \\
$\overline{\mathfrak{G}}$ & 54 & $1: 44$ & $0: 09$ \\
$\tilde{\mathfrak{G}}$ & 44 & $1: 32$ & $0: 13$ \\
$\Delta$ & 42 & $1: 31$ & $0: 16$ \\
$\mathrm{BSV}$ & 35 & $1: 10$ & $0: 21$ \\
$\Gamma_{3}$ & 75 & $1: 46$ & $0: 04$ \\
\hline
\end{tabular}

\begin{tabular}{cccc}
\hline & & \multicolumn{2}{c}{ Time (h:min) for } \\
\cline { 3 - 4 }$G$ & $c$ & $G / \gamma_{c+1} G$ & $M_{c+1}(G)$ \\
\hline$\Gamma_{4}$ & 71 & $1: 50$ & $0: 07$ \\
$\Gamma_{5}$ & 55 & $1: 40$ & $0: 04$ \\
$\Gamma_{7}$ & 46 & $1: 40$ & $0: 03$ \\
$\Gamma_{8}$ & 56 & $1: 54$ & $0: 06$ \\
$\Gamma_{9}$ & 61 & $1: 44$ & $0: 06$ \\
$\Gamma_{11}$ & 35 & $1: 54$ & $0: 02$ \\
\hline
\end{tabular}


In [21], Lysënok determined a first $L$-presentation for the group $\mathfrak{G}$; see Example 2. Even though it had already been proposed in $[\mathbf{1 4}]$ that the Grigorchuk group $\mathfrak{G}$ is not finitely presentable, a proof was not established until Grigorchuk explicitly computed in [15] the Schur multiplier of $\mathfrak{G}$ : it turned out to be infinitely generated 2-elementary abelian. We have computed the Dwyer quotients $M_{c}(\mathfrak{G})$ for $1 \leqslant c \leqslant 301$. These quotients are 2-elementary abelian with 2-ranks

$$
1,2,3^{[3]}, 5^{[6]}, 7^{[12]}, 9^{[24]}, 11^{[48]}, 13^{[96]}, 15^{[110]}
$$

This suggests the following conjecture.

Conjecture A. The Grigorchuk group $\mathfrak{G}$ satisfies

$$
M_{c}(\mathfrak{G}) \cong\left\{\begin{array}{cl}
\mathbb{Z}_{2} \text { or }\left(\mathbb{Z}_{2}\right)^{2} & \text { if } c=1 \text { or } 2, \text { respectively, } \\
\left(\mathbb{Z}_{2}\right)^{2 m+3} & \text { if } c \in\left\{3 \cdot 2^{m}, \ldots, 3 \cdot 2^{m+1}-1\right\} \text { with } m \in \mathbb{N}_{0} .
\end{array}\right.
$$

Further experiments suggest that the Schur multiplier of the Grigorchuk group $\mathfrak{G}$ has a $\{\sigma\}^{*}$-module structure, as given by Lemma 4 , of the form $\mathbb{Z}_{2} \oplus\left(\mathbb{Z}_{2}[\sigma]\right)^{2}$ where $\sigma$ fixes the first component.

The twisted twin $\overline{\mathfrak{G}}$ of the Grigorchuk group was introduced in [4]. It is invariantly finitely $L$-presented by

$$
\left\langle\{a, b, c, d\}\left|\left\{a^{2}, b^{2}, c^{2}, d^{2}\right\}\right|\{\bar{\sigma}\} \mid\left\{\left[d^{a}, d\right],\left[d, c^{a} b\right],\left[d,\left(c^{a} b\right)^{c}\right],\left[d,\left(c^{a} b\right)^{c}\right],\left[c^{a} b, c b^{a}\right]\right\}\right\rangle,
$$

where $\tilde{\sigma}$ is the free group endomorphism induced by the mapping

$$
\tilde{\sigma}:\left\{\begin{array}{l}
a \mapsto c^{a}, \\
b \mapsto d, \\
c \mapsto b^{a}, \\
d \mapsto c .
\end{array}\right.
$$

We have computed the Dwyer quotients $M_{c}(\overline{\mathfrak{G}})$ for $1 \leqslant c \leqslant 144$. These quotients are 2-elementary abelian with 2-ranks

$$
2,5,7,8^{[2]}, 11^{[2]}, 12^{[4]}, 15^{[4]}, 16^{[8]}, 19^{[8]}, 20^{[16]}, 23^{[16]}, 24^{[32]}, 27^{[32]}, 28^{[17]} .
$$

This suggests the following conjecture.

Conjecture B. The twisted twin $\overline{\mathfrak{G}}$ of the Grigorchuk group satisfies

$$
M_{c}(\overline{\mathfrak{G}}) \cong\left\{\begin{array}{cl}
\left(\mathbb{Z}_{2}\right)^{2},\left(\mathbb{Z}_{2}\right)^{5} \text { or }\left(\mathbb{Z}_{2}\right)^{7} & \text { if } c=1,2 \text { or } 3, \text { respectively, } \\
\left(\mathbb{Z}_{2}\right)^{4(m+1)+4} & \text { if } c \in\left\{2^{m+2}, \ldots, 2^{m+2}+2^{m+1}-1\right\} \text { with } m \in \mathbb{N}_{0}, \\
\left(\mathbb{Z}_{2}\right)^{4(m+1)+7} & \text { if } c \in\left\{2^{m+2}+2^{m+1}, \ldots, 2^{m+3}-1\right\} \text { with } m \in \mathbb{N}_{0} .
\end{array}\right.
$$

Further experiments suggest that the Schur multiplier of $\overline{\mathfrak{G}}$ has a $\{\bar{\sigma}\}^{*}$-module structure, as given by Lemma 4 , of the form $(\underset{\tilde{\mathscr{C}}}{\mathbb{Z}}[\bar{\sigma}])^{4}$; for a proof see [4].

The Grigorchuk super-group $\tilde{\mathfrak{G}}$ was introduced in [3]. It contains the Grigorchuk group $\mathfrak{G}$ as an infinite-index subgroup and is another example of a group with an intermediate word-growth. In [1], it was shown that $\tilde{\mathfrak{G}}$ admits the invariant finite $L$-presentation

$$
\left\langle\{\tilde{a}, \tilde{b}, \tilde{c}, \tilde{d}\}|\emptyset|\{\tilde{\sigma}\} \mid\left\{a^{2},[\tilde{b}, \tilde{c}],\left[\tilde{c}, \tilde{c}^{a}\right],\left[\tilde{c}, \tilde{d}^{a}\right],\left[\tilde{d}, \tilde{d}^{a}\right],\left[\tilde{c}^{a \tilde{b}},\left(\tilde{c}^{a \tilde{b}}\right)^{a}\right],\left[\tilde{c}^{a \tilde{b}},\left(\tilde{d}^{a \tilde{b}}\right)^{a}\right],\left[\tilde{d}^{a \tilde{b}},\left(\tilde{d}^{a \tilde{b}}\right)^{a}\right]\right\}\right\rangle
$$


where $\tilde{\sigma}$ is the free group endomorphism induced by the mapping

$$
\tilde{\sigma}:\left\{\begin{array}{l}
a \mapsto a \tilde{b} a, \\
\tilde{b} \mapsto \tilde{d}, \\
\tilde{c} \mapsto \tilde{b}, \\
\tilde{d} \mapsto \tilde{c} .
\end{array}\right.
$$

The Schur multiplier of the group $\tilde{\mathfrak{G}}$ is still unknown. We have computed the Dwyer quotients $M_{c}(\tilde{\mathfrak{G}})$ for $1 \leqslant c \leqslant 232$. These quotients are 2-elementary abelian with 2-ranks

$$
3,6,7,9^{[2]}, 11^{[2]}, 13^{[4]}, 15^{[4]}, 17^{[8]}, 19^{[8]}, 21^{[16]}, 23^{[16]}, 25^{[32]}, 27^{[32]}, 29^{[64]}, 31^{[41]} .
$$

This suggests the following conjecture.

Conjecture C. The Grigorchuk super-group $\tilde{\mathfrak{G}}$ satisfies

$$
M_{c}(\tilde{\mathfrak{G}}) \cong\left\{\begin{array}{cl}
\left(\mathbb{Z}_{2}\right)^{3},\left(\mathbb{Z}_{2}\right)^{6} \text { or }\left(\mathbb{Z}_{2}\right)^{7} & \text { if } c=1,2 \text { or } 3, \text { respectively, } \\
\left(\mathbb{Z}_{2}\right)^{4 m+5} & \text { if } c \in\left\{2 \cdot 2^{m}, \ldots, 3 \cdot 2^{m}-1\right\} \text { with } m \in \mathbb{N}, \\
\left(\mathbb{Z}_{2}\right)^{4 m+7} & \text { if } c \in\left\{3 \cdot 2^{m}, \ldots, 2 \cdot 2^{m+1}-1\right\} \text { with } m \in \mathbb{N} .
\end{array}\right.
$$

Further experiments suggest that the Schur multiplier of the Grigorchuk super-group has a $\{\tilde{\sigma}\}^{*}$-module structure, as given by Lemma 4 , of the form $\left(\mathbb{Z}_{2}\right)^{3} \oplus\left(\mathbb{Z}_{2}[\tilde{\sigma}]\right)^{4}$ where $\tilde{\sigma}$ cyclically permutes the first component.

The Basilica group $\Delta$ was introduced in $[\mathbf{1 7}, \mathbf{1 8}]$ as a torsion-free group defined by a three-state automaton. Bartholdi and Virág [5] computed the following invariant finite $L$-presentation:

$$
\Delta \cong\left\langle\{a, b\}|\emptyset|\{\sigma\} \mid\left\{\left[a, a^{b}\right]\right\}\right\rangle
$$

where $\sigma$ is the free group endomorphism induced by the mapping

$$
\sigma:\left\{\begin{array}{l}
a \mapsto b^{2}, \\
b \mapsto a .
\end{array}\right.
$$

We have computed the Dwyer quotients $M_{c}(\Delta)$ for $1 \leqslant c \leqslant 103$. These quotients are described by the following conjecture.

Conjecture D. The Basilica group $\Delta$ satisfies

$$
M_{c}(\Delta) \cong \mathbb{Z}^{2} \oplus \bigoplus_{\ell \in \mathbb{N}} \mathcal{A}_{\ell}(c) \quad \text { for each } c \geqslant 2
$$

where the groups $\mathcal{A}_{\ell}(c)$ are given by

$$
\mathcal{A}_{1}(c)=\left\{\begin{array}{cl}
0 & \text { if } c \in\{1, \ldots, 5\} \\
\mathbb{Z}_{2^{2(m+1)}} & \text { if } c \in\{2 m+6,2 m+7\}
\end{array}\right.
$$

and, for $\ell \geq 2$,

$$
\mathcal{A}_{\ell}(c)=\left\{\begin{array}{cl}
0 & \text { if } c \in\left\{1, \ldots, 3 \cdot 2^{\ell+1}-1\right\}, \\
\mathbb{Z}_{2^{2 m+1}} & \text { if } c \in\left\{(3+m) \cdot 2^{\ell+1}, \ldots,(3+m) \cdot 2^{\ell+1}+2^{\ell-1}-1\right\} \text { with } m \in \mathbb{N}_{0}, \\
\mathbb{Z}_{2^{2 m+2}} & \text { if } c \in\left\{(3+m) \cdot 2^{\ell+1}+2^{\ell-1}, \ldots,(4+m) \cdot 2^{\ell+1}-1\right\} \text { with } m \in \mathbb{N}_{0} .
\end{array}\right.
$$

Hence, the Basilica group $\Delta$ is not finitely presentable.

The Brunner-Sidki-Vieira group BSV was introduced in [9] as a just-non-solvable, torsionfree group acting on the binary tree. The authors also gave the following invariant finite $L$-presentation:

$$
\mathrm{BSV} \cong\left\langle\{a, b\}|\emptyset|\{\varepsilon\} \mid\left\{\left[b, b^{a}\right],\left[b, b^{a^{3}}\right]\right\}\right\rangle
$$


where $\varepsilon$ is the free group endomorphism induced by the mapping

$$
\varepsilon:\left\{\begin{array}{l}
a \mapsto a^{2}, \\
b \mapsto a^{2} b^{-1} a^{2} .
\end{array}\right.
$$

We have computed the Dwyer quotients $M_{c}(\mathrm{BSV})$ for $1 \leqslant c \leqslant 53$. These quotients are described by the following conjecture.

Conjecture E. The Brunner-Sidki-Vieira group BSV satisfies

$$
M_{c}(\mathrm{BSV}) \cong \mathbb{Z}^{2} \oplus \mathcal{A}(c) \oplus \bigoplus_{\ell \in \mathbb{N}} \mathcal{B}_{\ell}(c) \oplus \bigoplus_{\ell \in \mathbb{N}} \mathcal{C}_{\ell}(c) \quad \text { for each } c \geqslant 2,
$$

where the groups $\mathcal{A}(c), \mathcal{B}_{\ell}(c)$ and $\mathcal{C}_{\ell}(c)$ are given by

and, for $\ell \in \mathbb{N}$,

$$
\mathcal{A}(c)=\left\{\begin{array}{cl}
0 & \text { if } c \in\{1, \ldots, 3\} \\
\mathbb{Z}_{2^{2 m+1}} & \text { if } c \in\{2 m+4,2 m+5\} \text { with } m \in \mathbb{N}_{0}
\end{array}\right.
$$

$$
\begin{aligned}
& \mathcal{B}_{\ell}(c)=\left\{\begin{array}{cl}
0 & \text { if } c \in\left\{1, \ldots, 5 \cdot 2^{\ell-1}-1\right\}, \\
\mathbb{Z}_{2^{4 m+1}} & \text { if } c \in\left\{2^{\ell+2} m+5 \cdot 2^{\ell-1}, \ldots, 2^{\ell+2} m+6 \cdot 2^{\ell-1}-1\right\} \text { with } m \in \mathbb{N}_{0}, \\
\mathbb{Z}_{2^{4 m+2}} & \text { if } c \in\left\{2^{\ell+2} m+6 \cdot 2^{\ell-1}, \ldots, 2^{\ell+2} m+10 \cdot 2^{\ell-1}-1\right\} \text { with } m \in \mathbb{N}_{0}, \\
\mathbb{Z}_{2^{4 m+4}} & \text { if } c \in\left\{2^{\ell+2} m+10 \cdot 2^{\ell-1}, \ldots, 2^{\ell+2} m+13 \cdot 2^{\ell-1}-1\right\} \text { with } m \in \mathbb{N}_{0},
\end{array}\right. \\
& \mathcal{C}_{\ell}(c)=\left\{\begin{array}{cl}
0 & \text { if } c \in\left\{1, \ldots, 9 \cdot 2^{\ell-1}-1\right\}, \\
\mathbb{Z}_{2^{4 m+1}} & \text { if } c \in\left\{2^{\ell+2} m+9 \cdot 2^{\ell-1}, \ldots, 2^{\ell+2} m+12 \cdot 2^{\ell-1}-1\right\} \text { with } m \in \mathbb{N}_{0}, \\
\mathbb{Z}_{2^{4 m+2}} & \text { if } c \in\left\{2^{\ell+2} m+12 \cdot 2^{\ell-1}, \ldots, 2^{\ell+2} m+14 \cdot 2^{\ell-1}-1\right\} \text { with } m \in \mathbb{N}_{0}, \\
\mathbb{Z}_{2^{4 m+3}} & \text { if } c \in\left\{2^{\ell+2} m+14 \cdot 2^{\ell-1}, \ldots, 2^{\ell+2} m+16 \cdot 2^{\ell-1}-1\right\} \text { with } m \in \mathbb{N}_{0}, \\
\mathbb{Z}_{2^{4 m+4}} & \text { if } c \in\left\{2^{\ell+2} m+16 \cdot 2^{\ell-1}, \ldots, 2^{\ell+2} m+17 \cdot 2^{\ell-1}-1\right\} \text { with } m \in \mathbb{N}_{0} .
\end{array}\right.
\end{aligned}
$$

Hence, the Brunner-Sidki-Vieira group BSV is not finitely presentable.

\subsection{Dwyer quotients of some Fabrykowski-Gupta groups}

The Fabrykowski-Gupta group $\Gamma_{3}$ was introduced in $[\mathbf{1 1}]$ as an example of a group with an intermediate word-growth. For every positive integer $d$, Grigorchuk [16] described a generalization $\Gamma_{d}$ of the Fabrykowski-Gupta group $\Gamma_{3}$. A rather long invariant finite $L$-presentation was computed in [2]. Further, it was shown that if $d$ is not a prime power, the group $\Gamma_{d}$ has a maximal nilpotent quotient. This quotient is isomorphic to the maximal nilpotent quotient of the wreath product $\mathbb{Z}_{d} \prec \mathbb{Z}_{d}$. We therefore consider only those groups $\Gamma_{d}$ which admit a 'rich' lower central series, that is, for which the index $d$ is a prime power.

Let $d \in\{3,5,7,11\}$ be a prime. Then the Dwyer quotients $M_{c}\left(\Gamma_{d}\right)$ are $d$-elementary abelian with $d$-ranks as given in the following table.

\begin{tabular}{cc}
\hline$d$ & $\operatorname{rk}\left(M_{c}\left(\Gamma_{d}\right)\right)$ \\
\hline 3 & $0^{[2]}, 1^{[3]}, 2^{[0]}, 3^{[9]}, 4^{[1]}, 5^{[26]}, 6^{[4]}, 7^{[77]}, 8^{[13]}, 9^{[12]}$ \\
5 & $0^{[1]}, 1^{[4]}, 2^{[2]}, 3^{[20]}, 4^{[10]}, 5^{[100]} 6^{[2]}$ \\
7 & $0^{[1]}, 1^{[2]}, 2^{[6]}, 3^{[2]}, 4^{[14]}, 5^{[42]}, 6^{[14]}, 7^{[34]}$ \\
11 & $0^{[1]}, 1^{[2]}, 2^{[2]}, 3^{[2]}, 4^{[10]}, 5^{[2]}, 6^{[22]}, 7^{[22]}, 8^{[22]}, 9^{[27]}$ \\
\hline
\end{tabular}

As noted by Bartholdi and Siegenthaler, there is a pattern in the ranks of the Dwyer quotients $M_{c}\left(\Gamma_{d}\right)$. For example, it may be that

$$
M_{c}\left(\Gamma_{5}\right) \cong\left\{\begin{array}{cl}
0 & \text { if } c=0, \\
\mathbb{Z}_{5}^{2 m+1} & \text { if } c \in\left\{2+\frac{3}{2}\left(5^{m}-1\right), \ldots, 1+\frac{3}{2}\left(5^{m}-1\right)+4 \cdot 5^{m}\right\} \text { with } m \in \mathbb{N}_{0}, \\
\mathbb{Z}_{5}^{2 m+2} & \text { if } c \in\left\{2+\frac{3}{2}\left(5^{m}-1\right)+4 \cdot 5^{m}, \ldots, 1+\frac{3}{2}\left(5^{m+1}-1\right)\right\} \text { with } m \in \mathbb{N}_{0},
\end{array}\right.
$$

which suggests the following conjecture. 
Conjecture F. Let $d$ be a prime. Then the Schur multiplier of $\Gamma_{d}$, modulo the Dwyer kernel, is infinitely generated $d$-elementary abelian.

Finally, we summarize our results for $M_{c}\left(\Gamma_{d}\right)$ with $d \in\{4,8,9\}$. The abelian invariants of the Dwyer quotients $M_{c}\left(\Gamma_{d}\right)$ are as follows.

\begin{tabular}{|c|c|}
\hline$d$ & $M_{c}\left(\Gamma_{d}\right)$ \\
\hline 4 & $\begin{array}{c}(1)^{[1]}(2)^{[1]}(2,2)^{[1]}(2,4)^{[4]}(2,2,2,4)^{[1]} \\
(2,2,2,2,4)^{[4]}(2,2,2,4,4)^{[16]}(2,2,2,2,4,4)^{[1]}(2,2,2,2,2,4,4)^{[3]} \\
(2,2,2,2,2,2,4,4)^{[16]}(2,2,2,2,2,4,4,4)^{[64]}(2,2,2,2,2,2,4,4,4)^{[5]} \\
(2,2,2,2,2,2,2,4,4,4)^{[11]}(2,2,2,2,2,2,2,2,4,4,4)^{[26]}\end{array}$ \\
\hline 8 & $\begin{array}{c}(1)^{[1]}(8)^{[2]}(4,8)^{[3]}(2,4,8)^{[4]}(2,8,8)^{[1]}(2,2,8,8)^{[2]} \\
(2,2,2,8,8)^{[2]}(2,2,4,8,8)^{[2]}(2,4,4,8,8)^{[2]}(2,4,8,8,8)^{[2]} \\
(2,8,8,8,8)^{[8]}(2,2,8,8,8,8)^{[4]}(2,4,8,8,8,8)^{[20]}(2,2,4,8,8,8,8)^{[32]} \\
(2,2,8,8,8,8,8)^{[7]}(2,2,2,8,8,8,8,8)^{[16]}(2,2,2,2,8,8,8,8,8)^{[16]} \\
(2,2,2,4,8,8,8,8,8)^{[16]}(2,2,4,4,8,8,8,8,8)^{[3]}\end{array}$ \\
\hline 9 & $\begin{array}{c}(1)^{[1]}(9)^{[2]}(3,9)^{[2]}(3,3,9)^{[4]}(3,9,9)^{[2]} \\
(9,9,9)^{[2]}(3,9,9,9)^{[2]}(3,3,9,9,9)^{[4]}(3,9,9,9,9)^{[2]} \\
(9,9,9,9,9)^{[12]}(3,9,9,9,9,9)^{[18]}(3,3,9,9,9,9,9)^{[36]} \\
(3,9,9,9,9,9,9)^{[18]}(9,9,9,9,9,9,9)^{[17]}(3,9,9,9,9,9,9,9)^{[12]}\end{array}$ \\
\hline
\end{tabular}

Again, these computational results suggest that the groups $\Gamma_{d}$ are not finitely presentable. Further, the exponent of $M_{c}\left(\Gamma_{d}\right)$ is most likely the index $d$ itself.

Acknowledgements. I would like to thank Bettina Eick, Laurent Bartholdi and Olivier Siegenthaler for many helpful discussions. I am grateful to the referee for valuable comments and suggestions.

\section{References}

1. L. BARThOldi, 'Endomorphic presentations of branch groups', J. Algebra 268 (2003) no. 2, 419-443.

2. L. Bartholdi, B. Eick and R. Hartung, 'A nilpotent quotient algorithm for certain infinitely presented groups and its applications', Internat. J. Algebra Comput. 18 (2008) no. 8, 1321-1344.

3. L. BARTholdi and R. I. GRigorchuk, 'On parabolic subgroups and Hecke algebras of some fractal groups', Serdica Math. J. 28 (2002) no. 1, 47-90.

4. L. Bartholdi and O. Siegenthaler, 'The twisted twin of the Grigorchuk group', Internat. J. Algebra Comput. 24 (2010) no. 4, 465-488.

5. L. BARTholdi and B. VirÁG, 'Amenability via random walks', Duke Math. J. 130 (2005) no. 1, 39-56.

6. G. Baumslag, Topics in combinatorial group theory,, Lectures in Mathematics, ETH Zürich (Birkhäuser, Basel, 1993).

7. N. Blackburn and L. Evens, 'Schur multipliers of p-groups', J. reine angew. Math. 309 (1979) 100-113.

8. V. V. Bludov, 'On residually torsion-free nilpotent groups', J. Group Theory 12 (2009) no. 4, 579-590.

9. A. M. Brunner, S. Sidki and A. C. Vieira, 'A just nonsolvable torsion-free group defined on the binary tree', J. Algebra 211 (1999) no. 1, 99-114.

10. B. EICK and W. NiCKEL, 'Computing the Schur multiplicator and the nonabelian tensor square of a polycyclic group', J. Algebra 320 (2008) no. 2, 927-944.

11. J. FAbrYkowski and N. Gupta, 'On groups with sub-exponential growth functions', J. Indian Math. Soc. (N.S.) 49 (1987) no. 3-4, 249-256, 1985.

12. The GAP group, 'GAP — groups, algorithms and programming, version 4.4.10', 2007.

13. C. M. GoRdon, 'Some embedding theorems and undecidability questions for groups', Combinatorial and geometric group theory (Edinburgh, 1993), London Mathematical Society Lecture Note Series 204 (Cambridge University Press, Cambridge, 1995) 105-110. 
14. R. I. Grigorchuk, 'On Burnside's problem on periodic groups', Funktsional. Anal. i Prilozhen. 14 (1980) no. $1,53-54$

15. R. I. Grigorchuk, 'On the system of defining relations and the Schur multiplier of periodic groups generated by finite automata', Groups St Andrews 1997 in Bath, I, London Mathematical Society Lecture Note Series 260 (Cambridge University Press, Cambridge, 1999) 290-317.

16. R. I. Grigorchuk, 'Just infinite branch groups', New horizons in pro- $p$ groups,, Progress in Mathematics 184 (Birkhäuser, Boston, MA, 2000) 121-179.

17. R. I. GRIGORChUK and A. ŻUK, 'On a torsion-free weakly branch group defined by a three state automaton', Internat. J. Algebra Comput. 12 (2002) no. 1-2, 223-246. International Conference on Geometric and Combinatorial Methods in Group Theory and Semigroup Theory (Lincoln, NE, 2000).

18. R. I. Grigorchuk and A. ŻUK, 'Spectral properties of a torsion-free weakly branch group defined by a three state automaton', Computational and statistical group theory: AMS special sessions on geometric group theory (Las Vegas, NV, 2001) and computational group theory, Hoboken, NJ, 2001,, Contemporary Mathematics 298 (American Mathematical Society, Providence, RI, 2002) 57-82.

19. R. HARTUNG, 'NQL-nilpotent quotients of $L$-presented groups', a refereed GAP 4 package, 2009; see [12].

20. D. F. Holt, 'The calculation of the Schur multiplier of a permutation group', Computational group theory, Durham, 1982, (Academic Press, London, 1984) 307-319.

21. I. G. LYSËNOK, 'A set of defining relations for the Grigorchuk group', Mat. Zametki 38 (1985) no. 4, 503-516.

22. R. V. MikhaĬLov, 'Residual nilpotence and residual solvability of groups', Mat. Sb. 196 (2005) no. 11, 109-126.

23. W. Nickel, 'Computing nilpotent quotients of finitely presented groups', Geometric and computational perspectives on infinite groups: Proceedings of a joint DIMACS/Geometry Center workshop, Minneapolis, MN and New Brunswick, NJ, 1994,, DIMACS Series in Discrete Mathematics and Theoretical Computer Science 25 (American Mathematical Society, Providence, RI, 1996) 175-191.

24. D. J. S. Robinson, A course in the theory of groups,, 2nd edn, Graduate Texts in Mathematics 80 (Springer, New York, 1996).

25. V. Sergiescu, 'A quick introduction to Burnside's problem', Group theory from a geometrical viewpoint, Trieste, 1990, (World Scientific Publishers, River Edge, NJ, 1991) 622-629.

René Hartung

Institute of Computational

Mathematics

University of Braunschweig

Pockelsstraße 14, 38106 Braunschweig

Germany

r.hartung@tu-bs.de 